\title{
Enhanced Energy Harvesting Ability of ZnO/PAN Hybrid Piezoelectric Nanogenerators
}

DOI:

10.1021/acsami.0c14490

\section{Document Version}

Accepted author manuscript

Link to publication record in Manchester Research Explorer

\section{Citation for published version (APA):}

Sun, Y., Liu, Y., Zheng, Y., Li, Z., Fan, J., Wang, L., Liu, X., Liu, J., \& Shou, W. (2020). Enhanced Energy Harvesting Ability of ZnO/PAN Hybrid Piezoelectric Nanogenerators. ACS Applied Materials and Interfaces, 12(49), 54936-54945. https://doi.org/10.1021/acsami.0c14490

\section{Published in:}

ACS Applied Materials and Interfaces

\section{Citing this paper}

Please note that where the full-text provided on Manchester Research Explorer is the Author Accepted Manuscript or Proof version this may differ from the final Published version. If citing, it is advised that you check and use the publisher's definitive version.

\section{General rights}

Copyright and moral rights for the publications made accessible in the Research Explorer are retained by the authors and/or other copyright owners and it is a condition of accessing publications that users recognise and abide by the legal requirements associated with these rights.

\section{Takedown policy}

If you believe that this document breaches copyright please refer to the University of Manchester's Takedown Procedures [http://man.ac.uk/04Y6Bo] or contact uml.scholarlycommunications@manchester.ac.uk providing relevant details, so we can investigate your claim.

\section{OPEN ACCESS}




\title{
Enhanced Energy Harvesting Ability of ZnO/PAN Hybrid Piezoelectric Nanogenerator
}

Yue Sun, Yong Liu*, Yide Zheng, Zongjie Li, Jie Fan, Liang Wang, Xuqing Liu*, Jian Liu, Wan Shou*

Y. Sun, Prof. Y. Liu, Y. Zheng, Dr. Z. Li, Prof. J. Fan, Dr. L. Wang

State Key Laboratory of Separation Membranes and membrane Processes, School of Textile science and engineering, Tiangong University, Tianjin 300387, China

Dr. X. Liu

Department of Materials, University of Manchester, Oxford Road, Manchester M13 9PL, UK

Dr. J. Liu

School of Textiles, 495 Fenghua Road, Zhejiang Fashion Institute of Technology, Ningbo, Zhejiang Province 315000, China

Dr. W. Shou

Computer Science and Artificial Intelligence Lab (CSAIL), Electrical Engineering and Computer Science Department, Massachusetts Institute of Technology Cambridge, MA 02139, USA

\begin{abstract}
Miniaturization of energy conversion and storage devices has attracted remarkable consideration in the application of wearable electronics. Compared with film-based flexible electronics, fiber-based wearable electronics (e.g., nanogenerator and sensors made from electrospun nanofiber), are more appealing and promising for wearables. However, there are two bottlenecks, low power output, and poor sensing capability, limiting the application of piezoelectric nanofibers. Herein, we integrated zinc oxide
\end{abstract}


nanorods (ZnO NRs) to a less known piezoelectric polymer, Polyacrylonitrile (PAN) nanofiber, forming a $\mathrm{ZnO} / \mathrm{PAN}$ nano-fabric, which significantly improved the pressure sensitivity and vibrational energy harvesting ability by about 2.7 times compared with the pristine PAN nanofiber, and the maximum output power density of $\sim 10.8 \mathrm{~mW} \cdot \mathrm{m}^{-2}$ is achieved. Noteworthy, the $\mathrm{ZnO} / \mathrm{PAN}$ nano-fabric showed a power output about twice of the one made of $\mathrm{ZnO}$ and polyvinylidene fluride (PVDF). It was revealed that the integration of $\mathrm{ZnO}$ NRs clearly improved the planar zig-zag conformation in microstructures of PAN nanofiber. Further, successful demonstrations of a mechanically robust pressure sensor and wearable power source confirms the potential applications in human activity monitoring and personal thermal management, respectively.

KEYWORDS: piezoelectric nanogenerator, nanofiber, ZnO nanorods, wearable electronics, nanofabric, Energy Harvesting

\section{INTRODUCTION}

With the coming era of the Internet of Things (IoT), there is an increasing need for wearable electronics, and the advancement of wearable electronics is largely dependent on the ability of power source. Self-powering micro-electronics, ${ }^{1-4}$ especially piezoelectric nanogenerators (PENG), are emerging as the main sustainable energy scavenging wearable devices that effectively convert mechanical energy into electric energy. ${ }^{5-7}$ As an essential part of the PENG, various piezoelectric materials, have been investigated, including $\mathrm{ZnO},{ }^{8} \mathrm{PZT},{ }^{9}$ polymer, ${ }^{10}$ composites $^{11,}{ }^{12}$, peptide, ${ }^{13}$ etc . Among these, polymer and polymer composites are especially popular for wearable electronics due to their relatively large piezoelectric coefficient and flexibility, such as polyvinylidene fluoride (PVDF), which is a widely used semi-crystalline piezoelectric polymer. Recently, polyacrylonitrile (PAN) was rediscovered as a novel piezoelectric polymer material for energy harvesting, to replace polyvinylidene 
fluoride (PVDF). The electrospun PAN nanofiber membrane showed larger piezoelectric output, smaller dielectric loss, higher thermal stability and lower price and toxicity compared to PVDF membrane. ${ }^{14}$

However, the electromechanical conversion capability of the electrospun PAN piezoelectric film is still low, and it is challenging to fabricate high-efficiency power generating devices. Furthermore, the surface of the PAN nanofiber membrane has a certain amount of charge residue due to stretching and polarization under a high voltage electrostatic field. These residual surface charges dissipate over time, which could seriously affect the stability of the piezoelectric output of the nanofiber membrane. Therefore, improving the piezoelectric energy conversion efficiency of the PAN nanofiber membrane and reducing the residual charge on the surface plays an important in the fabrication of efficient and stable energy harvesting devices.

It is known that the conformation of the macromolecular chain plays a determined role in the piezoelectricity of polymer piezoelectric materials. ${ }^{15}$ In solid-state, there are two conformations in the PAN macromolecular chain, namely, $3^{1}$-helical and planar zig-zag, ${ }^{16},{ }^{17}$ which endows the PAN with piezoelectricity. To improve the conformation, several methods such as mechanical stretching, ${ }^{18}$ electric field polarization, ${ }^{19}$ and material recombination ${ }^{20}$ were explored. Among them, the piezoelectric composites formed by inorganic and polymeric piezoelectric materials can not only improve the conformation of piezoelectric polymers but also endow them excellent properties of inorganic piezoelectric materials, broadening the application range of piezoelectric nanogenerators. ${ }^{21,22}$ Typically, $\mathrm{ZnO}$ nanocomposites are widely used to enhance the piezoelectric performances. ${ }^{23,}{ }^{24}$ It was found the combination of $\mathrm{ZnO}$ nanomaterials and PVDF can greatly increase the content of $\beta$ crystal phase in PVDF, resulting in the enhancement of the electromechanical coupling coefficient of piezoelectric polymers. ${ }^{25-}$ ${ }^{27}$ Generally, $\mathrm{ZnO}$ based nanocomposites showed promising piezoelectric performance and flexibility as flexible electronics. ${ }^{28,29}$

We herein report a flexible PENG based on a nano-fabric that constituted of PAN nanofibers and $\mathrm{ZnO}$ nanorods (NRs) fabricated by electrospinning and low-temperature hydrothermal growth. It is 
different from the studies of improving the ferroelectric phase content by adding metals, carbon nanotubes, and semiconductors, which improve piezoelectricity by reducing charge transfer resistance in piezoelectric devices or increasing electroactive substances in devices. In this work, through the in-situ nano-stretching force of $\mathrm{ZnO} \mathrm{NRs}$ during hydrothermal growth, the macromolecular chain segments in PAN nanofibers are fully stretched to achieve the purpose of increasing the content of the planar zig-zag conformation. The growth of $\mathrm{ZnO}$ NRs can improve greatly the piezoelectricity of $\mathrm{ZnO} / \mathrm{PAN}$-based nano-fabric PENG. The use of the synergistic piezoelectric effect of both $\mathrm{ZnO}$ NRs and PAN enables PENG to have higher electrical output performance. Through conformation engineering, we boosted its piezoelectric performance by 2.7 times by hydrothermal synthesis of $\mathrm{ZnO}$ NRs into PAN nanofibers. Compared with common $\mathrm{ZnO} / \mathrm{PVDF}$ piezoelectric nano-fabrics, the dpiezoelectric properties is twice that of $\mathrm{ZnO} / \mathrm{PAN}$ nano-fabrics. Further, we demonstrate that the developed ZnO/PAN-based nano-fabric can be used as a robust sensor for human activity monitoring and energy source for personal thermal management.

\section{RESULTS AND DISCUSSION}

A facile and efficient method combining electrospinning and low-temperature hydrothermal synthesis technology was used to fabricate the $\mathrm{ZnO} / \mathrm{PAN}$-based piezoelectric nano-fabric. ${ }^{30}$ As illustrated in Figure $1, \mathrm{Zn}(\mathrm{Ac})_{2} / \mathrm{PAN}$ nanofiber membrane is first fabricated through electrospinning (details can be found in the experimental section). Afterward, the $\mathrm{Zn}(\mathrm{Ac})_{2}$ in the nanofiber membrane is decomposed into $\mathrm{ZnO}$ seeds by heat treatment, which is used to synthesize $\mathrm{ZnO}$ NRs in the growth solution. Finally, the nano-fabric is sandwiched between two current collectors for energy harvesting. 


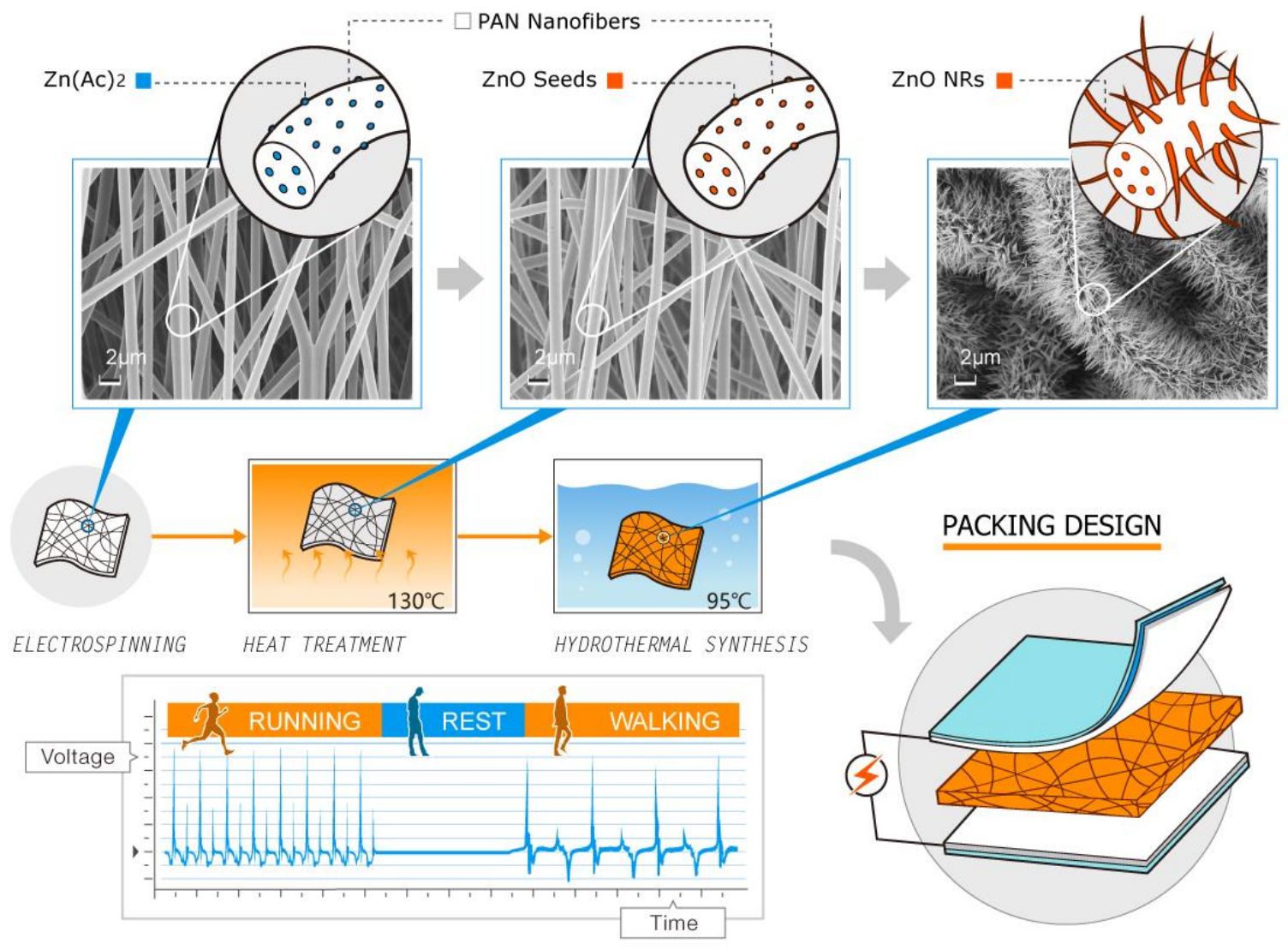

Figure 1. Schematic diagram of the fabrication process of the $\mathrm{ZnO} / \mathrm{PAN}$-based nano-fabric for PENG.

The morphology of the electrospun PAN nanofiber is shown in Figure 2a, with a diameter of $650 \pm$ $50 \mathrm{~nm}$. The pure PAN nanofibers with uniform thickness and smooth morphology can be prepared by electrospinning technology. For $\mathrm{ZnO} / \mathrm{PAN}$ nano-fabric, the $\mathrm{ZnO}$ NRs show a uniform structure (Figure 2b) with an average length of about $2.1 \mu \mathrm{m}$ and a diameter of about $98 \mathrm{~nm}$ after heat treatment and hydrothermal growth (Figure S1). More details can be found in the Experimental Section for fabrication and characterization. The insert in Figure $2 \mathrm{~b}$ is the cross section of $\mathrm{ZnO} / \mathrm{PAN}$ nano-fabric, showing $\mathrm{ZnO}$ NRs grew well on the side of nanofiber membrane, which immersed in the growth solution during the hydrothermal process, while there were not $\mathrm{ZnO}$ NRs on the other side. The asymmetric structure of composite membrane ensures high electrical output. Furthermore, Energy-dispersive X-ray spectroscopy (EDS) mapping (as shown in Figure 2c) clearly reveals that the zinc and oxygen elements are uniformly distributed on the nanofiber. The Fourier transform infrared spectroscopy (FTIR) spectra in Figure 2d 
shows the vibration bands of planar zig-zag conformation and $3^{1}$-helical conformation at $1250 \mathrm{~cm}^{-1}$ and $1230 \mathrm{~cm}^{-1}$, respectively. ${ }^{31}$ It can be noticed that PAN powder, PAN nanofibers and $\mathrm{ZnO} / \mathrm{PAN}$ nano-fabric all possess similar FTIR spectral curves, but with different content of planar zig-zag conformation, which can be calculated by the following equation (1), ${ }^{14}$

$$
\Phi=\frac{S_{1250}}{S_{1250}+S_{1230}}
$$

where $S_{1230}$ and $S_{1250}$ represent the peak area at $1230 \mathrm{~cm}^{-1}$ and $1250 \mathrm{~cm}^{-1}$, respectively. ZnO/PAN nanofabric has a $\Phi$ of $51.55 \%$, which is higher than the PAN nanofiber membrane $(\Phi=45.59 \%)$, and PAN powder $(\Phi=31.56 \%)$ (more detailed comparison can be found in Figures S2 and S3).
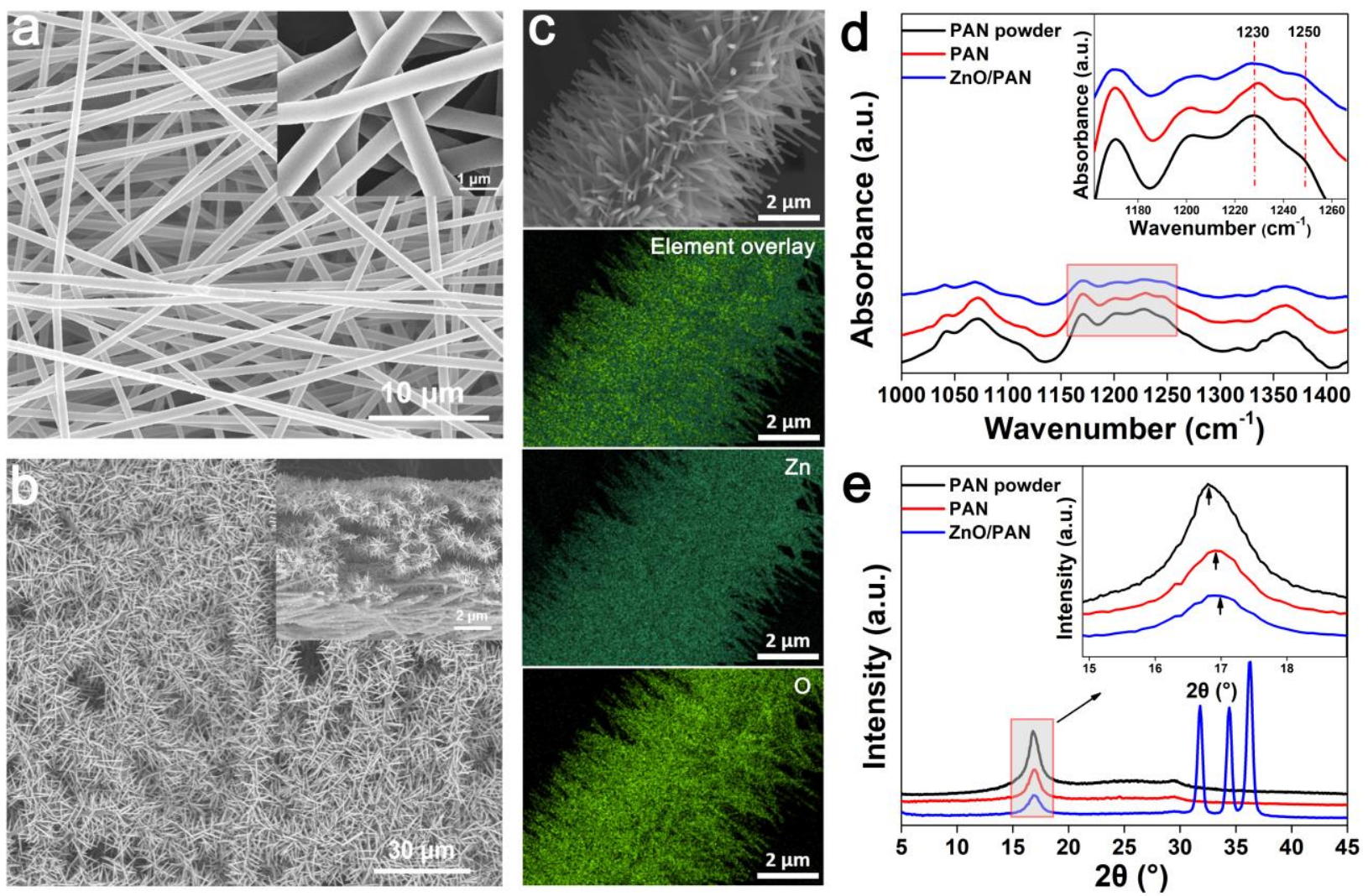

Figure 2. Characterization of PAN nanofiber membrane and $\mathrm{ZnO} / \mathrm{PAN}$ nano-fabric. SEM images of (a) PAN nanofibers and (b) ZnO/PAN nano-fabric (inset: corresponding cross-section), (c) a single $\mathrm{ZnO} / \mathrm{PAN}$ nanofiber and corresponding EDS mapping. (d) FTIR spectra of PAN powder, PAN nanofiber membrane and $\mathrm{ZnO} / \mathrm{PAN}$ nano-fabric. (e) XRD patterns of PAN powder, PAN nanofiber membrane and $\mathrm{ZnO} / \mathrm{PAN}$ nano-fabric. 
The XRD patterns further prove the difference in the molecular conformation of PAN powder, PAN nanofiber membrane and $\mathrm{ZnO} / \mathrm{PAN}$ nano-fabric. As shown in Figure 2e, their diffraction peaks at $2 \theta=$ $16.82^{\circ}, 16.94^{\circ}, 17.02^{\circ}$ correspond to the crystallization peaks of PAN ${ }^{32}$ In $\mathrm{ZnO} / \mathrm{PAN}$ nano-fabric, XRD pattern shows significant peaks at $2 \theta=32.0^{\circ}, 34.4^{\circ}, 36.2^{\circ}$, corresponding to (100), (002), (101) crystal faces of the $\mathrm{ZnO}$ hexagonal wurtzite structure. ${ }^{22}$ The content of planar zig-zag conformation in PAN can be measured by $2 \theta-17 .{ }^{33}$ Figure 2e shows ZnO/PAN nano-fabric has the highest content of planar zigzag conformation, followed by the PAN nanofiber membrane and PAN powder (the specific values can be found in Figure S4). Furthermore, a significant shift at the $17^{\circ}$ peak indicates a change in the (100) interplanar spacing, which also indicates the content of planar zig-zag conformation. ${ }^{34}$ The interplanar spacing $(d)$ can be calculated by the Bragg equation $(2),{ }^{35}$

$$
2 d \sin \theta=n \lambda
$$

where $d$ is the interplanar spacing of the corresponding crystal faces, $\theta$ and $\lambda$ are the diffraction angle and $\mathrm{X}$-ray wavelength. It is calculated that the $d$ of PAN powder, PAN nanofiber membrane and ZnO/PAN nano-fabric decrease successively, which are $0.531,0.524$, and $0.519 \mathrm{~nm}$, respectively (Figure S5). Both FTIR and XRD patterns demonstrated that the growth of ZnO NRs on PAN nanofibers could increase the content of planar zig-zag conformation. 

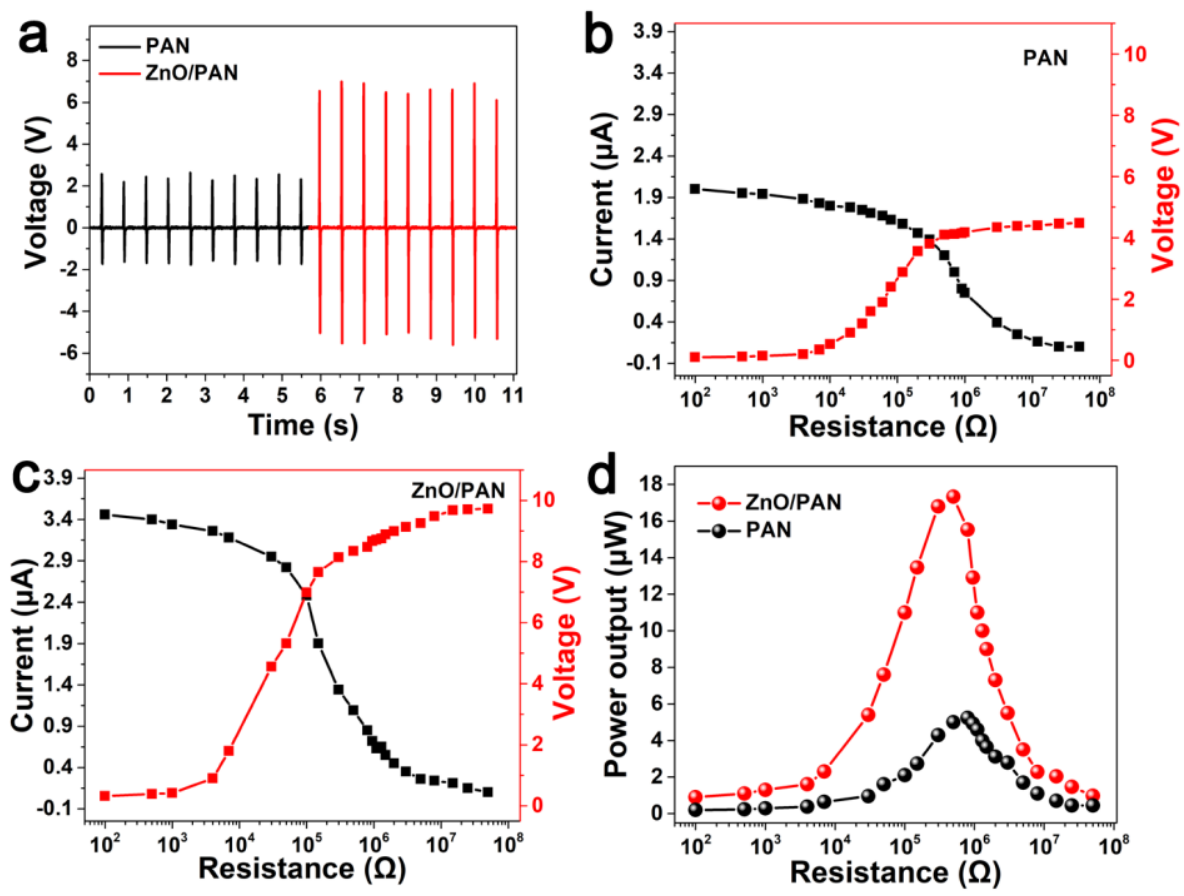

Figure 3. Piezoelectric performance of PAN nanofiber and $\mathrm{ZnO} / \mathrm{PAN}$ nano-fabric. (a) Piezoelectric output voltage. The influence of the external load resistance on peak voltage and current (b) PAN and (c) $\mathrm{ZnO} / \mathrm{PAN}$. (d) The influence of external load resistance on the power output. (All the samples were measured with a frequency of $2 \mathrm{~Hz}$, and impact force of $8 \mathrm{~N}$ ).

The output voltages from PAN and $\mathrm{ZnO} / \mathrm{PAN}$ nano-fabric are presented in Figure 3a, under continuous compression impact $(8 \mathrm{~N})$ with a frequency of $\sim 2 \mathrm{~Hz}$. The output voltage of $\mathrm{ZnO} / \mathrm{PAN}$-based PENG is $\sim 6.5 \mathrm{~V}$, which is significantly higher than that obtained from PAN-based PENG is $(\sim 2.4 \mathrm{~V})$. Similarly, their output currents also show a big difference, with values of 2.3 and $1.0 \mu \mathrm{A}$, respectively, for $\mathrm{ZnO} / \mathrm{PAN}$ and PAN (see Figure S6). There are three possible reasons for the high voltage and current of $\mathrm{ZnO} / \mathrm{PAN}$ nano-fabric. ${ }^{28,29}$ Firstly, the growth of $\mathrm{ZnO}$ NRs increases the planar zig-zag conformation content in PAN, which makes the PAN nanofiber membrane dominated by the helical conformation to the zig-zag conformation, resulting in the improvement of piezoelectric properties of PAN nanofiber membrane itself. Secondly, under the compression impact of the same force, ZnO NRs might cause the PAN nanofibers to produce greater deformation, resulting in a larger signal output of the PAN piezoelectric nanofiber membrane. Finally, $\mathrm{ZnO}$, as a piezoelectric material, has its intrinsic piezoelectric properties. The synergistic piezoelectric effect of the two piezoelectric materials presents better 
piezoelectric properties than a single material. To prove that $\mathrm{ZnO}$ NRs growth can promote the formation of plane zig-zag conformation, we doped $\mathrm{ZnO}$ nanoparticles to prepare $\mathrm{ZnO} / \mathrm{PAN}$ nanofiber membrane for characterization (Figure S7). The results showed that direct doping of $\mathrm{ZnO}$ hardly improves the content of planar zig-zag conformation in PAN (see in Figure S7a, b). Although the piezoelectricity of the nanofiber membrane prepared by doping $\mathrm{ZnO}$ nanoparticles was improved compared with pure PAN (Figure S7c, d), it was still lower than that of nanofiber membrane with grown $\mathrm{ZnO}$ NRs. Therefore, the growth of $\mathrm{ZnO}$ NRs did play an important role in improving the piezoelectricity of the nano-fabric.

When the external pressing force was applied to ZnO/PAN-based nano-fabric PENG, the ZnO NRs grown radially along with the fiber first undergo bending deformation, producing a positive potential on the stretched side and a negative potential on the compressed side. ${ }^{36}$ And then, the force is further transferred to the PAN nanofibers to produce the corresponding positive and negative potentials. When the force is released, the $\mathrm{ZnO}$ NRs and PAN strain disappear at the same time. Thus, a compressive impact on the hybrid structure can generate a pulse signal, which can be used for energy harvester and touch sensor. The enlarged view of single-cycle voltage output under pressing and releasing is shown in Figure S8. The effect of external load resistance on the electric outputs of the PAN and ZnO/PAN-based nano-fabric PENG is shown in Figure $3 b$ and c. Generally, the output current decreases with increasing the external resistance; however, the output voltage has an opposite trend. As shown in Figure 3d, the maximum output power density of the PAN-based PENG is $3.2 \mathrm{~mW} \mathrm{~m}^{-2}$ when the external resistance is $800 \mathrm{k} \Omega$; while the $\mathrm{ZnO} / \mathrm{PAN}$-based nano-fabric PENG has a maximum output power density of $10.8 \mathrm{~mW}$ $\mathrm{m}^{-2}$ with an external resistance of $700 \mathrm{~K} \Omega$. Compare with PAN-based PENG, the ZnO/PAN-based nanofabric PENG has a much higher maximum power output, more than 6 times. 

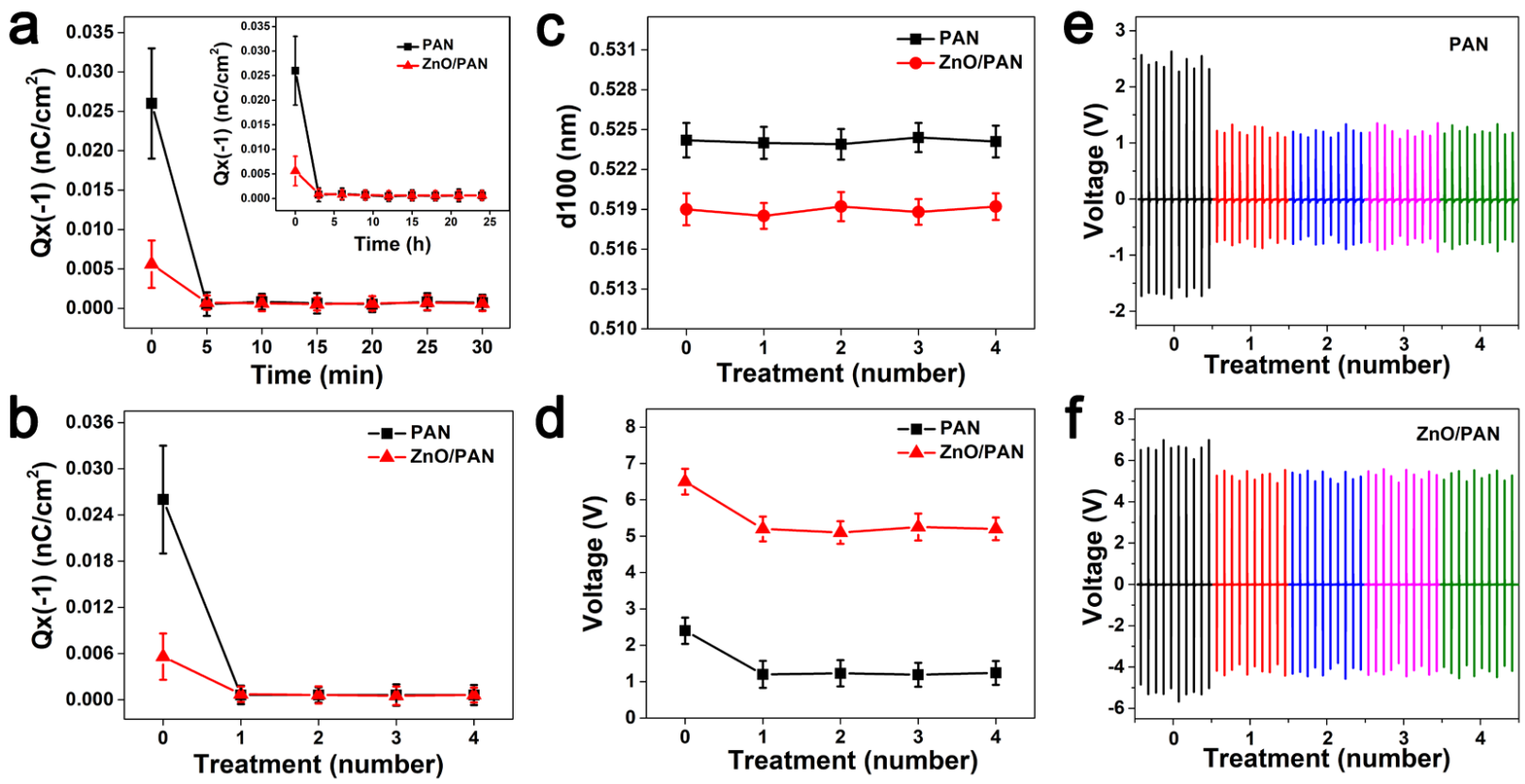

Figure 4. (a) The change of residual charge with isopropanol soaking time and drying time in PAN, $\mathrm{ZnO} / \mathrm{PAN}$ nano-fabric. The change of residual charge (b), plane spacing (c), and voltage output (d) with isopropanol treatment number in PAN nanofiber membranes, $\mathrm{ZnO} / \mathrm{PAN}$ nano-fabric. The voltage outputs of PAN nanofiber membrane (e), ZnO/PAN nano-fabric (f) before and after repeated charge removal treatment (working area $16 \mathrm{~cm}^{2}$, membrane thickness $100 \mu \mathrm{m}$, frequency $2 \mathrm{~Hz}$, impact force $8 \mathrm{~N}$ ).

Further, we found that the residual charge in the $\mathrm{ZnO} / \mathrm{PAN}$ piezoelectric nano-fabric has little effect on the electromechanical conversion performance (as shown in Figure 4a). The ZnO/PAN nano-fabric carried charges of about $0.0056 \mathrm{nC} \cdot \mathrm{cm}^{-2}$, which is much lower than that carried by the PAN nanofiber membrane (about $0.026 \mathrm{nC} \cdot \mathrm{cm}^{-2}$ ). This difference is mainly caused by the hydrothermal growth of $\mathrm{ZnO}$ NRs, where the charges dissipated in the solution. After soaking in isopropanol (IPA) for 30 minutes, the residual charge in the PAN nanofiber membrane and ZnO/PAN nano-fabric both decreased significantly and only maintained at around $0.00124 \mathrm{nC} \cdot \mathrm{cm}^{-2}$. With further drying, the residual charge decreased from $0.00124 \mathrm{nC} \cdot \mathrm{cm}^{-2}$ to $0.000625 \mathrm{nC} \cdot \mathrm{cm}^{-2}$ after $24 \mathrm{~h}$. To ensure complete elimination of residual charge, charge removal treatment was repeated four times (as shown in Figure 4b); however, no further decrease was observed, which means the first treatment had almost completely removed the residual charge. Also, no clear change is observed in the interplanar spacing (and the conformation) of PAN nanofiber membrane and $\mathrm{ZnO} / \mathrm{PAN}$ nano-fabric (as indicated in Figure 4c). Therefore, the reduction of their output 
voltage is mainly attributed to the removal of residual charge (Figure 4d). In addition, Figure 4e and $\mathrm{f}$ show that, after the removal of the residual charges, the output voltage of the ZnO/PAN nano-fabric maintains at $81 \%$, while the PAN nanofiber membrane drops to $49 \%$ of the original value. These results reveal that $\mathrm{ZnO} / \mathrm{PAN}$ nano-fabric has good electrical stability.
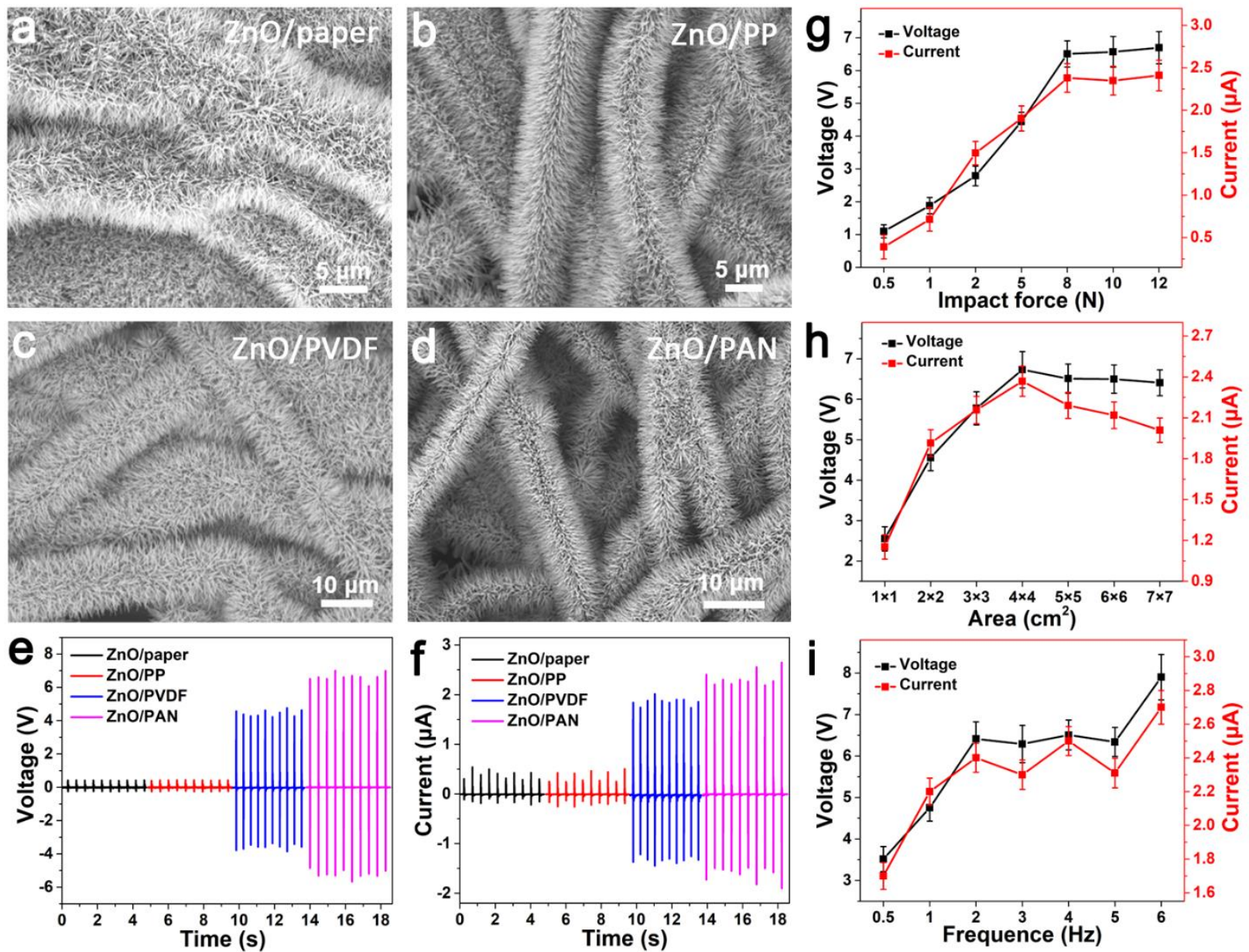

Figure 5. The SEM image of ZnO NRs on different substrates, (a) paper, (b) PP, (c) PVDF, and (d) PAN. The comparison of voltage outputs (e) and currents (f) of four different substrates (working area $16 \mathrm{~cm}^{2}$, membrane thickness $100 \mu \mathrm{m}$, frequency $2 \mathrm{~Hz}$, impact force $\left.8 \mathrm{~N}\right)$. The influence of $(\mathrm{g})$ impact force, (h) nano-fabric size, and (i) impact frequency on the piezoelectric performance of $\mathrm{ZnO} / \mathrm{PAN}$-based piezoelectric nanogenerator. (g, working area $16 \mathrm{~cm}^{2}$, frequency $2 \mathrm{~Hz}$; h, impact force $8 \mathrm{~N}$, frequency 2 $\mathrm{Hz}$; i, working area $16 \mathrm{~cm}^{2}$, impact force $8 \mathrm{~N}$. All the membranes have a thickness of $100 \mu \mathrm{m}$.)

To investigate the influence of flexible substrates on the piezoelectric performance, four nanocomposites were fabricated and examined. The surface morphology of four piezoelectric nanocomposites are shown in Figure 5a-d, ZnO NRs uniformly grew successfully on paper, PP, PVDF, 
and PAN substrates without significant difference. Figure 5e and f show that the ZnO/PAN nano-fabric has the highest piezoelectric performance, whose output voltage and current are $51.16 \%$ and $27.78 \%$ higher than the values of $\mathrm{ZnO} / \mathrm{PVDF}$ nano-fabric $(4.3 \mathrm{~V}$ and $1.8 \mu \mathrm{A})$, respectively; the other two common substrates without piezoelectricity show much lower output voltage and current (less than $0.5 \mathrm{~V}$ and 0.4 $\mu \mathrm{A})$. Furthermore, we calculated the output power density of the four PENGs made of $\mathrm{ZnO} / \mathrm{paper}$, $\mathrm{ZnO} / \mathrm{PP}, \mathrm{ZnO} / \mathrm{PVDF}$ and $\mathrm{ZnO} / \mathrm{PAN}$, and they are $0.08,0.07,4.8$ and $9.5 \mathrm{~mW} \mathrm{~m}^{-2}$, respectively. It is noteworthy that the $\mathrm{ZnO} / \mathrm{PAN}$-based nano-fabric PENG outperforms the piezoelectric performance of $\mathrm{ZnO} / \mathrm{PVDF}$-based nano-fabric PENG by about twice. The output power is much larger than most reported $\mathrm{ZnO} \mathrm{NWs},{ }^{37-39} \mathrm{PVDF},{ }^{40,}{ }^{41} \mathrm{PAN}^{14}$ and PVDF-ZnO NWs. ${ }^{24}$ It is believed that the excellent piezoelectric performance results from the synergistic effect of $\mathrm{ZnO}$ NRs and PAN nanofibers. With further optimization of the configuration and packaging process, the power can be even higher (More detailed comparison can be found in Table S1).

Additionally, we investigated the electrical output performances of the $\mathrm{ZnO} / \mathrm{PAN}$-based nano-fabric PENG under different impact forces, areas, and frequencies, respectively. Figure $5 \mathrm{~g}$ shows the output voltage and current of the device first increases with impact force (until $8 \mathrm{~N}$ ) and then stabilizes around $6.51 \mathrm{~V}$ and $2.38 \mu \mathrm{A}$, respectively. It is found that when the area increases from 1 to $16 \mathrm{~cm}^{2}$ (Figure $5 \mathrm{~h}$ ), the output voltage significantly increases from 2.55 to $6.73 \mathrm{~V}$, and the current also increased from 1.15 to $2.36 \mu \mathrm{A}$ (frequency $2 \mathrm{~Hz}$, impact force $8 \mathrm{~N}$ ). However, the output voltage and current decrease slightly when the area further increases. Initially, the output voltage and current increase with the increase of the working area, which can be attributed to the increase of the working area leading to an increase in active material, thereby increasing the charge output. However, as the working area further increases, the resistance to charge transfering from the hybrid film to the wire increases. Under the same compression force, the increase of the working area can also increase the internal consumption of the piezoelectric device, ${ }^{14}$ which in turn affects the electrical properties of the hybrid film. As expected, the output electrical signals increase with the frequency in the range of 0.5 to $2 \mathrm{~Hz}$, as shown in Figure 5i, and then 
the output electrical signals became stable (between 2 to $5 \mathrm{~Hz}$ ). A further increase was observed when the frequency increased to $6 \mathrm{~Hz}$. It is inferred that the recovery of incomplete deformation due to the fast load and unload might be the main cause of stable section (from 2 to $5 \mathrm{~Hz}$ ); while the speed is high enough (at $6 \mathrm{~Hz}$ ), the nano-fabric can be stressed further to generate higher voltage and current. The detailed corresponding output electrical signals can be found in Figure S9-S11. 

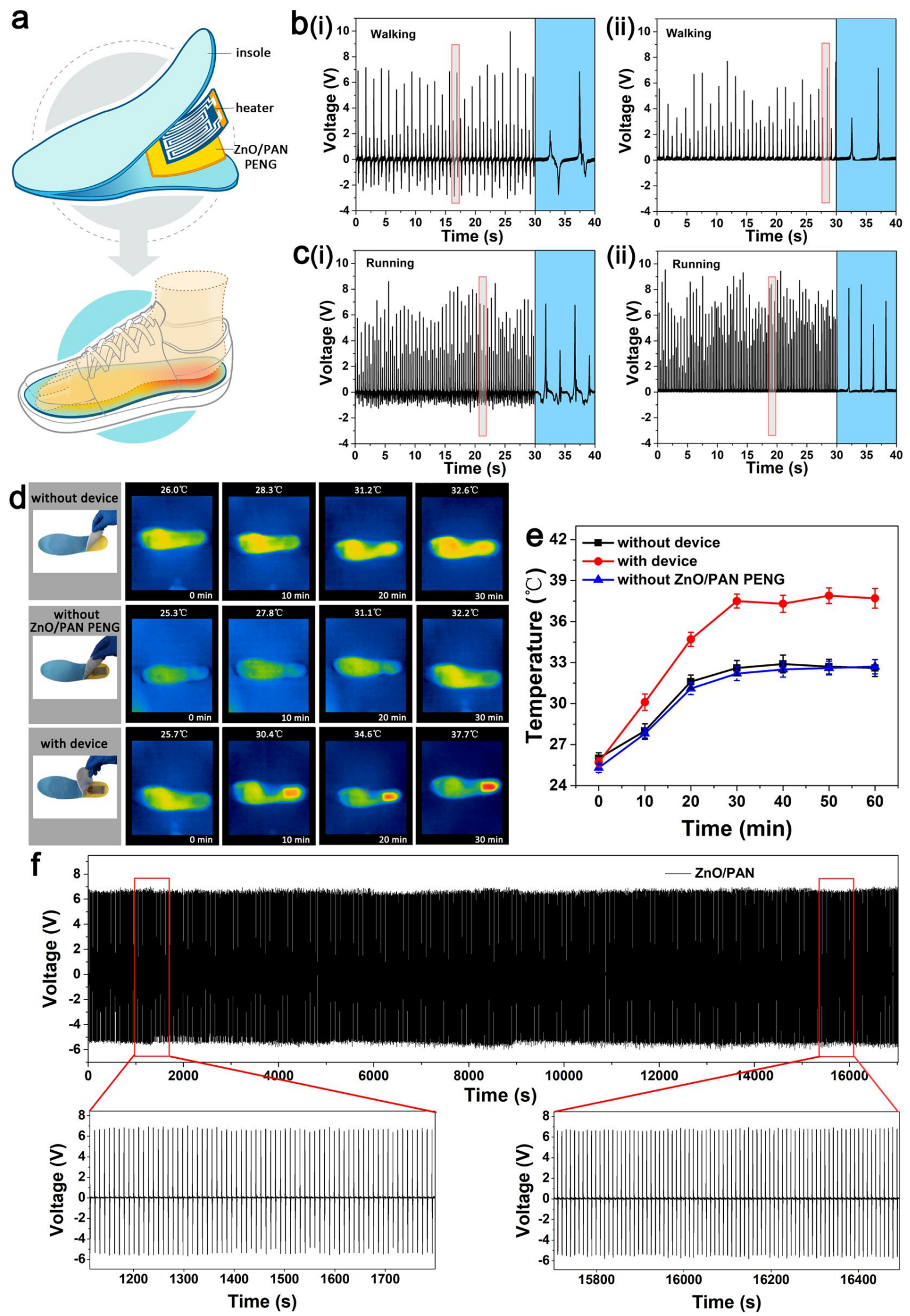
Figure 6. Application of the electric energy generated by the PENG to drive a commercial electric heating sheet. (a) The schematic drawing of self-heating insole. (b) The output voltage generated the $\mathrm{ZnO} / \mathrm{PAN}$-based nano-fabric PENG during walking (i) raw data and (ii) rectified data. (c) The output voltage generated the $\mathrm{ZnO} / \mathrm{PAN}$-based nano-fabric PENG during running (i) raw data and (ii) rectified data. (d) Digital photo and IR images of the insole. (e) Dependence of the surface temperature of three insoles with different movement times. (f) Load and unload cycle test of the ZnO/PAN nano-fabric (insets show the stable performance at the beginning and end of the test).

Finally, we integrated the ZnO/PAN-based nano-fabric PENG with a sheet heater $(0.2 \mathrm{~W})$ through a rectifier bridge and embedded them into a smart insole, as illustrated in Figure 6a. Two practical applications were demonstrated using the fabricated nano-fabric. First, the nano-fabric was used as a pressure sensor, which can distinguish the activity between walking and running (as shown in Figure 6b and c). It can be seen that the maximum peak voltage during walking is about $7.36 \mathrm{~V}$ (rectified voltage $\sim 5.92 \mathrm{~V}$ ); while the maximum peak voltage during running is about $8.17 \mathrm{~V}$ (rectified voltage $\sim 6.16 \mathrm{~V}$ ) and also the voltage signal is denser. This is because the impact force during walking is smaller than that during running. It is also observed that the output voltage signal is unstable due to the irregular motion. Thus, based on the signal, we can tell whether the person is running or walking. Such nano-fabric can be used for other finer force detection as well. Second, we employed this nano-fabric as a wearable power source to power a heater for the warmth of foot (as shown in Figure 6d). With regular motion (walking), the surface temperature of the insole (with a self-powered heater) increased much faster than the one without a device (Figure 6e). After about $30 \mathrm{~min}$, the insole with a self-powered heater shows a stable temperature that is $5{ }^{\circ} \mathrm{C}$ higher than the one without the device. Compared with the oridinayr insole, the surface temperature of the insole with the heater (but without ZnO/PAN-based nano-fabric PENG) does not change significantly and has the same trend of change after walking. The results proved that the elevated temperature of the sheet heater was powered by $\mathrm{ZnO} / \mathrm{PAN}-$ based nano-fabric PENG. Thus, the nano-fabric-based PENG can be used for personal thermal management when in a combination of a heater or cooler. ${ }^{42}$ Furthermore, we prove that the piezoelectric nano-fabric has a robust mechanical performance, which can undergo repeated operation more than 1500 times without any degradation 
(Figure 6f). These results prove that the $\mathrm{ZnO} / \mathrm{PAN}-$ based nano-fabric PENG has great potential for human activity monitoring and personal thermal management.

\section{CONCLUSIONS}

In summary, we successfully enhanced the planar zig-zag conformation of PAN nanofiber by insitu growth of $\mathrm{ZnO} \mathrm{NRs}$, which showed a significant improvement ( 2.7 times) in piezoelectric performance compared with the pristine PAN nanofiber. It is found that the removal of residual charge has a negligible effect on its piezoelectric performance. The piezoelectric effect of $\mathrm{ZnO} / \mathrm{PAN}$ nano-fabric outperforms that made of $\mathrm{ZnO} / \mathrm{PVDF}$ by about twice, with an output power density of $9.5 \mathrm{~mW} \mathrm{~m}^{-2}$. We also demonstrated that the $\mathrm{ZnO} / \mathrm{PAN}$-based nano-fabric could work as a robust sensor to monitor human activity (walking and running). Meanwhile, the piezoelectric nano-fabric was also integrated with a sheet heater for personal thermal management, for example, foot warmth in winter. This work provides a higher piezoelectric nano-fabrics, which have broad applications, such as healthcare, personal thermal management, and human activity monitoring. ${ }^{28}$

\section{EXPERIMENTAL SECTION}

Materials. Polyacrylonitrile (PAN) powder (Mw, 1500,000) and poly(vinylidene fluoride) (PVDF) powder (Mw, 517,000) were purchased from Beijing JinBeiNuo Technology Co., Ltd. and Solvay. Zinc acetate $\left(\mathrm{Zn}(\mathrm{Ac})_{2}\right)$, zinc nitrate hexahydrate $\left(\mathrm{Zn}\left(\mathrm{NO}_{3}\right)_{2} \cdot 6 \mathrm{H}_{2} \mathrm{O}\right)$, hexamethylenetetramine $\left(\mathrm{C}_{6} \mathrm{H}_{12} \mathrm{~N}_{4}\right)$ and ammonium hydroxide $\left(\mathrm{NH}_{3} \cdot \mathrm{H}_{2} \mathrm{O}\right)$ were provided by Tianjin Fengchuan Chemical Reagent Technology Co., Ltd. N, N-dimethylformamide (DMF) ( $\geq 99 \%$ ), acetone and isopropanol (IPA) were obtained from Tianjin Kermel Chemical Reagent Co., Ltd. Polypropylene (PP) non-woven fabric was purchased from Changshu binteer non-woven fabric Co., Ltd. All reagents were used without further purification.

Electrospinning: First, an appropriate amount of $\mathrm{Zn}(\mathrm{Ac})_{2}$ was added to the mixture of DMF and acetone (volume ratio of DMF and acetone, 7:3) with magnetic stirring until completely dissolved. Then PAN 
solution $(18 \% \mathrm{w} / \mathrm{v})$ was prepared by adding PAN powder (mass ratio of PAN and $\left.\mathrm{Zn}(\mathrm{Ac})_{2}, 10: 1\right)$ to the above solution. The mixture was stirred at $50{ }^{\circ} \mathrm{C}$ for $10 \mathrm{~h}$, resulting in clear and homogeneous spinning solutions. A reference PAN nanofiber membrane was electrospun using pure PAN (18\% w/v) solution. $\mathrm{Zn}(\mathrm{Ac})_{2} / \mathrm{PVDF}$ solution $(20 \% \mathrm{w} / \mathrm{v})$ was prepared in the same way, heated and stirred at $90{ }^{\circ} \mathrm{C}$ for $8 \mathrm{~h}$. A metal needle-based electrospinning setup was employed to prepare $\mathrm{Zn}(\mathrm{Ac})_{2} / \mathrm{PAN}$ and $\mathrm{Zn}(\mathrm{Ac})_{2} / \mathrm{PVDF}$ nanofiber mats with a thickness of $\sim 100 \mu \mathrm{m}$. A high voltage of $22 \mathrm{kV}$ was applied by a DC power supply to the needle. Electrospinning was performed at a spinning solution flow rate of $0.5 \mathrm{~mL} \cdot \mathrm{h}^{-1}$, with a spinning distance of $15 \mathrm{~cm}$. Fibers were collected on a grounded collector (length $20 \mathrm{~cm}$; diameter $9 \mathrm{~cm}$ ) rotating at $1000 \mathrm{rpm}$.

Preparation of $\mathrm{ZnO}$ Seed Layer. The prepared nanofiber membrane was placed in a $130{ }^{\circ} \mathrm{C}$ oven for heat treatment for $24 \mathrm{~h}$ to decompose $\mathrm{Zn}(\mathrm{Ac})_{2}$ into $\mathrm{ZnO}$ nanoparticle seeds. For comparison, $\mathrm{ZnO}$ seed layer was prepared on the filter paper (thickness $\sim 150 \mu \mathrm{m}$ ) and polypropylene (PP) non-woven fabric (thickness $\sim 170 \mu \mathrm{m}$ ) after being cleaned using ultrasonic in acetone, ethanol and deionized water. $\mathrm{Zn}(\mathrm{Ac})_{2}$ was dissolved in isopropyl alcohol $(200 \mathrm{~mL})$ and stirred vigorously at $60^{\circ} \mathrm{C}$ for 20 min to obtain a solution $(10 \mathrm{mM})$. The filter paper and PP non-woven fabric were immersed in seed solution $(10 \mathrm{mM})$ for $20 \mathrm{~min}$ and then cured at $80{ }^{\circ} \mathrm{C}$ for $30 \mathrm{~min}$. This process was repeated 3 times. All samples prepared with the $\mathrm{ZnO}$ seed layer were used for the hydrothermal growth in the next process.

Growth of $\mathrm{ZnO}$ Nanorods. ZnO nanorod arrays were prepared by low-temperature hydrothermal synthesis technology. The growth solution consisted of $\mathrm{Zn}\left(\mathrm{NO}_{3}\right)_{2} \cdot 6 \mathrm{H}_{2} \mathrm{O}(30 \mathrm{mM})$, HMTA $(10 \mathrm{mM})$ and $\mathrm{NH}_{3} \cdot \mathrm{H}_{2} \mathrm{O}(5 \mathrm{~mL})$ in deionized water $(100 \mathrm{~mL})$. The prepared nanofiber membranes, filter paper and PP non-woven fabric with $\mathrm{ZnO}$ seed layer were vertically dipped into growth solution (400 mL), sealed and placed in $95^{\circ} \mathrm{C}$ oven for $3 \mathrm{~h}$. Finally, the samples with $\mathrm{ZnO}$ nanorods were rinsed with a large amount of running DI water, ultrasonically cleaned for 30 minutes to remove residue and then dried in air to obtain $\mathrm{ZnO} / \mathrm{PAN}, \mathrm{ZnO} / \mathrm{PVDF}, \mathrm{ZnO} /$ paper and $\mathrm{ZnO} / \mathrm{PP}$ nanofiber membranes. 
Fabrication of Hybrid Nanogenerators. A piece of $\mathrm{ZnO} / \mathrm{PAN}$ composite nanofiber membrane was sandwiched between two thin copper tapes serving as top and bottom electrodes of the hybrid nanogenerator. To protect from any external mechanical damage, the polyethylene terephthalates (PET) film with a thickness of $80 \mu \mathrm{m}$ was further covered on the device to make an encapsulation using a laminator. Typically, the hybrid nanogenerator had an effective area of $16 \mathrm{~cm}^{2}(4 \mathrm{~cm} \times 4 \mathrm{~cm})$. In order to compare the electric output performances, the hybrid structure piezoelectric devices based on ZnO/PVDF, $\mathrm{ZnO} /$ paper and $\mathrm{ZnO} / \mathrm{PP}$ non-woven was also made into the same size.

Measurement of Piezoelectric Properties. The energy-conversion properties of hybrid piezoelectric nanogenerators were measured by piezoelectric test device consists of a pressure applicator (manufactured by Changsha Nanoapparatus Co., Ltd. China) and an electrochemistry work station (eCorder 401). The fabricated energy conversion device was mounted on the base support to withstand the compressive force from the pressure applicator at the desired frequency. During the process of applying compressive force, the output voltage and current signals of the hybrid nanogenerators were recorded in real-time by an electrochemical work station. The details of the test system have been described in the previous work. ${ }^{14}$

Elimination of Residual Charges. Residual charges were removed from composite nanofiber membranes according to the method specified in the standard (EN 779:2012). The residual charge of nanofibers was measured using an Agilent 34401A meter.

Characterizations. The surface morphology of the $\mathrm{ZnO} / \mathrm{PAN}$ composite nanofiber membrane was characterized by scanning electron microscopy (SEM, Hitachi S4800). The diameter of nanofiber and $\mathrm{ZnO}$ nanorod and $\mathrm{ZnO}$ nanorod length were measured based on the SEM images using an image processing software Nano Measurer 1.2. Fourier transform infrared spectroscopy (FTIR) was recorded with an FTIR spectrophotometer (Nicolet iS50). The crystallographic structures and composition were confirmed by X-ray diffraction (XRD, ARL ADVANT'X IntelliPowerTM 4200) with Cu-Ka radiation 
operated at $40 \mathrm{kV}$ and $30 \mathrm{~mA}$. The thickness of the samples was measured by a thickness gauge. The Universal Testing Machine (INSTRON 5969, USA) applied different forces and frequencies to the membrane. The IR camera (FLIR TG165) was used to measure infrared thermal images at different temperatures produced by a commercial heater $(0.2 \mathrm{~W})$.

\section{ASSOCIATED CONTENT}

\section{Supporting Information}

The Supporting Information is available free of charge on the ACS Publications website.

Structure size of ZnO/PAN; FTIR analysis of PAN planar zig-zag conformation content; comparison of piezoelectric nanogenerators based on ZnO NWs/NRs and piezoelectric; $\Delta \theta(2 \theta-17)$ values and Interplanar spacing of different types of PAN based on XRD; the output currents of PAN and ZnO/PAN based nanogenerators; the voltage output enlarged view of one cycle under pressing and releasing; the output voltage and current of $\mathrm{ZnO} / \mathrm{PAN}$-based PENG with different impact forces, areas and frequencies (PDF).

\section{AUTHOR INFORMATION}

\section{Corresponding Authors}

*E-mail: $\underline{\text { liuyong@ tiangong.edu.cn; xuqing.liu@manchester.ac.uk; wanshou@ mit.edu. }}$

\section{Author Contributions}

The manuscript was written through contributions of all authors. All authors have given approval to the final version of the manuscript.

\section{Funding Sources}

National Natural Science Foundation of China (Grant No. 51573133), the China Postdoctoral Science Foundation Grant (2018M630276), Scientific Research Project of Tianjin Municipal Education Commission (No.2019ZD01), Natural Science Foundation of Ningbo (No. 2018A610104). 


\section{Notes}

The authors declare no competing financial interest.

\section{ACKNOWLEDGMENTS}

The authors gratefully acknowledge financial support by the National Natural Science Foundation of China (Grant No. 51573133), the China Postdoctoral Science Foundation Grant (2018M630276), Scientific Research Project of Tianjin Municipal Education Commission (No.2019ZD01) and Natural Science Foundation of Ningbo (No. 2018A610104).

\section{REFERENCES}

(1) Wang, Z.; Tan, L.; Pan, X.; Liu, G.; He, Y.; Jin, W.; Li, M.; Hu, Y.; Gu, H. Self-Powered Viscosity and Pressure Sensing in Microfluidic Systems Based on the Piezoelectric Energy Harvesting of Flowing Droplets. ACS Appl. Mater. Inter. 2017, 9, 28586-28595.

(2) Hosseini, E. S.; Manjakkal, L.; Shakthivel, D.; Dahiya, R. Glycine-Chitosan-Based Flexible Biodegradable Piezoelectric Pressure Sensor. ACS Appl. Mater. Inter. 2020, 12, 9008-9016.

(3) Zhao, L.; Li, H.; Meng, J.; Li, Z. The Recent Advances in Self-Powered Medical Information Sensors. InfoMat 2020, 2, 212-234.

(4) Zheng, Q.; Jin, Y.; Liu, Z.; Ouyang, H.; Li, H.; Shi, B.; Jiang, W.; Zhang, H.; Li, Z.; Wang, Z. L. Robust Multilayered Encapsulation for High-Performance Triboelectric Nanogenerator in Harsh Environment. ACS Applied Materials \& Interfaces 2016, 8, 26697-26703.

(5) Jiang, D.; Shi, B.; Ouyang, H.; Fan, Y.; Wang, Z. L.; Chen, Z.-M.; Li, Z. A 25-Year Bibliometric Study of Implantable Energy Harvesters and Self-Powered Implantable Medical Electronics Researches. Materials Today Energy 2020, 16, 100386. 
(6) Jiang, D.; Shi, B.; Ouyang, H.; Fan, Y.; Wang, Z. L.; Li, Z. Emerging Implantable Energy Harvesters and Self-Powered Implantable Medical Electronics. ACS Nano 2020, 14, 6436-6448.

(7) Li, Z.; Zheng, Q.; Wang, Z. L.; Li, Z. Nanogenerator-Based Self-Powered Sensors for Wearable and Implantable Electronics. Research 2020, 2020, 8710686.

(8) Bui, Q. C.; Ardila, G.; Sarigiannidou, E.; Roussel, H.; Jiménez, C.; Chaix-Pluchery, O.; Guerfi, Y.; Bassani, F.; Donatini, F.; Mescot, X.; Salem, B.; Consonni, V. Morphology Transition of ZnO from Thin Film to Nanowires on Silicon and Its Correlated Enhanced Zinc Polarity Uniformity and Piezoelectric Responses. ACS Appl. Mater. Inter. 2020, 12, 29583-29593.

(9) Qi, Y.; Kim, J.; Nguyen, T. D.; Lisko, B.; Purohit, P. K.; McAlpine, M. C. Enhanced Piezoelectricity and Stretchability in Energy Harvesting Devices Fabricated from Buckled PZT Ribbons. Nano Lett. 2011, 11, 1331-1336.

(10) Sun, J.; Yang, A.; Zhao, C.; Liu, F.; Li, Z. Recent Progress of Nanogenerators Acting as Biomedical Sensors in Vivo. Science Bulletin 2019, 64, 1336-1347.

(11) Chowdhury, A. R.; Abdullah, A. M.; Hussain, I.; Lopez, J.; Cantu, D.; Gupta, S. K.; Mao, Y.; Danti, S.; Uddin, M. J. Lithium Doped Zinc Oxide Based Flexible Piezoelectric-Triboelectric Hybrid Nanogenerator. Nano Energy 2019, 61, 327-336.

(12) Jiang, J.; Tu, S.; Fu, R.; Li, J.; Hu, F.; Yan, B.; Gu, Y.; Chen, S. Flexible Piezoelectric Pressure Tactile Sensor Based on Electrospun $\mathrm{BaTiO}_{3} / \mathrm{Poly}($ Vinylidene Fluoride) Nanocomposite Membrane. ACS Appl. Mater. Inter. 2020, 12, 33989-33998.

(13) Nguyen, V.; Zhu, R.; Jenkins, K.; Yang, R. Self-Assembly of Diphenylalanine Peptide with Controlled Polarization for Power Generation. Nat. Commun. 2016, 7, 1-6.

(14) Wang, W.; Zheng, Y.; Jin, X.; Sun, Y.; Lu, B.; Wang, H.; Fang, J.; Shao, H.; Lin, T. Unexpectedly High Piezoelectricity of Electrospun Polyacrylonitrile Nanofiber Membranes. Nano energy 2019, 56, 588-594.

(15) Kepler, R.; Anderson, R. Piezoelectricity in Polymers. Crit. Rev. Solid State 1980, 9, 399-447. 
(16) Rizzo, P.; Auriemma, F.; Guerra, G.; Petraccone, V.; Corradini, P. Conformational Disorder in the Pseudohexagonal Form of Atactic Polyacrylonitrile. Macromolecules 1996, 29, 8852-8861.

(17) Hobson, R. J.; Windle, A. H. Crystalline Structure of Atactic Polyacrylonitrile. Macromolecules 1993, 26, 6903-6907.

(18) Kim, G. H.; Hong, S. M.; Seo, Y. Piezoelectric Properties of Poly(Vinylidene Fluoride) and Carbon Nanotube Blends: B -Phase Development. Phys. Chem. Chem. Phys. 2009, 11, 10506-10512.

(19) Lei, T.; Yu, L.; Zheng, G.; Wang, L.; Wu, D.; Sun, D. Electrospinning-Induced Preferred Dipole Orientation in PVDF Fibers. J. Mater. Sci. 2015, 50, 4342-4347.

(20) Tiana, G.; Denga, W.; Gaoa, Y.; Xionga, D.; Yana, C. Rich Lamellar Crystal Baklava-Structured PZT/PVDF Piezoelectric Sensor toward Individual Table Tennis Training. Nano Energy 2019, 59, 574581.

(21) Li, J.; Zhao, C.; Xia, K.; Liu, X.; Li, D.; Han, J. Enhanced Piezoelectric Output of the PVDFTrFE/ZnO Flexible Piezoelectric Nanogenerator by Surface Modification. Appl. Surf. Sci. 2019, 463, 626-634.

(22) Thakur, P.; Kool, A.; Hoque, N. A.; Bagchi, B.; Khatun, F.; Biswas, P.; Brahma, D.; Roy, S.; Banerjee, S.; Das, S. Superior Performances of in Situ Synthesized ZnO/PVDF Thin Film Based SelfPoled Piezoelectric Nanogenerator and Self-Charged Photo-Power Bank with High Durability. Nano Energy 2018, 44, 456-467.

(23) Bafqi, M. S. S.; Bagherzadeh, R.; Latifi, M. Fabrication of Composite PVDF-ZnO Nanofiber Mats by Electrospinning for Energy Scavenging Application with Enhanced Efficiency. J. Polym. Res. 2015, $22,130$.

(24) Choi, M.; Murillo, G.; Hwang, S.; Kim, J. W.; Jung, J. H.; Chen, C.-Y.; Lee, M. Mechanical and Electrical Characterization of PVDF-ZnO Hybrid Structure for Application to Nanogenerator. Nano Energy 2017, 33, 462-468. 
(25) Singh, H. H.; Khare, N. Flexible ZnO-PVDF/PTFE Based Piezo-Tribo Hybrid Nanogenerator. Nano Energy 2018, 51, 216-222.

(26) Lee, S.; Bae, S. H.; Lin, L.; Yang, Y.; Park, C.; Kim, S. W.; Cha, S. N.; Kim, H.; Park, Y. J.; Wang, Z. L. Super - Flexible Nanogenerator for Energy Harvesting from Gentle Wind and as an Active Deformation Sensor. Advanced Functional Materials 2013, 23, 2445-2449.

(27) Baniasadi, M.; Xu, Z.; Hong, S.; Naraghi, M.; Minary-Jolandan, M. Thermo-Electromechanical Behavior of Piezoelectric Nanofibers. ACS Appl. Mater. Inter. 2016, 8, 2540-2551.

(28) Kim, M.; Wu, Y.; Kan, E.; Fan, J. Breathable and Flexible Piezoelectric ZnO@ PVDF Fibrous Nanogenerator for Wearable Applications. Polymers 2018, 10, 745.

(29) Li, Z.; Zhang, X.; Li, G. In Situ ZnO Nanowire Growth to Promote the PVDF Piezo Phase and the ZnO-PVDF Hybrid Self-Rectified Nanogenerator as a Touch Sensor. Phys. Chem. Chem. Phys. 2014, $16,5475-5479$.

(30) Pan, T. D.; Li, Z. J.; Shou, D. H.; Shou, W.; Fan, J. T.; Liu, X.; Liu, Y. Buoyancy Assisted Janus Membrane Preparation by ZnO Interfacial Deposition for Water Pollution Treatment and Self Cleaning. Adv. Mater. Interfaces 2019, 6, 1901130.

(31) Minagawa, M.; Miyano, K.; Takahashi, M.; Yoshii, F. Infrared Characteristic Absorption Bands of Highly Isotactic Poly(Acrylonitrile). Macromolecules 1988, 21, 2387-2391.

(32) Wang, Z. Y.; Su, K. H.; Fan, H. Q.; Wen, Z. Y. Possible Reasons That Piezoelectricity Has Not Been Found in Bulk Polymer of Polyvinylidene Cyanide. Polymer 2008, 49, 2542-2547.

(33) Minagawa, M.; Taira, T.; Yabuta, Y.; Nozaki, K.; Yoshii, F. An Anomalous Tacticity-Crystallinity Relationship: A Waxd Study of Stereoregular Isotactic (83-25) Poly(Acrylonitrile) Powder Prepared by Urea Clathrate Polymerization. Macromolecules 2001, 34, 3679-3683.

(34) Hu, X.; Johnson, D. J.; Tomka, J. G. Molecular Modelling of the Structure of Polyacrylonitrile Fibres. Journal of the Textile Institute Proceedings \& Abstracts 1995, 86, 322-331. 
(35) Newman, R. H. Simulation of X-Ray Diffractograms Relevant to the Purported Polymorphs Cellulose IV I and IV II. Cellulose 2008, 15, 769-778.

(36) Park, H. K.; Lee, K. Y.; Seo, J. S.; Jeong, J. A.; Kim, H. K.; Choi, D.; Kim, S. W. Charge Generating Mode Control in High - Performance Transparent Flexible Piezoelectric Nanogenerators. Adv. Funct. Mater. 2011, 21, 1187-1193.

(37) Xu, S.; Qin, Y.; Xu, C.; Wei, Y.; Yang, R.; Wang, Z. L. Self-Powered Nanowire Devices. Nature nanotechnology 2010, 5, 366-373.

(38) Lee, M.; Bae, J.; Lee, J.; Lee, C.-S.; Hong, S.; Wang, Z. L. Self-Powered Environmental Sensor System Driven by Nanogenerators. Energ. Environ. Sci. 2011, 4, 3359-3363.

(39) Fan, F. R.; Tang, W.; Wang, Z. L. Flexible Nanogenerators for Energy Harvesting and Self Powered Electronics. Adv. Mater. 2016, 28, 4283-4305.

(40) Fang, J.; Wang, X.; Lin, T. Electrical Power Generator from Randomly Oriented Electrospun Poly (Vinylidene Fluoride) Nanofibre Membranes. J. Mater. Chem. 2011, 21, 11088-11091.

(41) Fuh, Y.-K.; Ho, H.-C.; Wang, B.-S.; Li, S.-C. All-Fiber Transparent Piezoelectric Harvester with a Cooperatively Enhanced Structure. Nanotechnology 2016, 27, 435403.

(42) Fang, J.; Niu, H.; Wang, H.; Wang, X.; Lin, T. Enhanced Mechanical Energy Harvesting Using Needleless Electrospun Poly (Vinylidene Fluoride) Nanofibre Webs. Energ. Environ. Sci. 2013, 6, 21962202. 
For Table of Contents Only

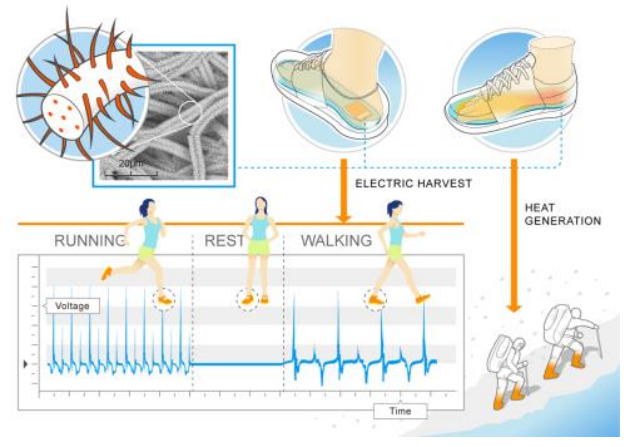

